\title{
Variation altitudinale de la distribution des plantes à activité insecticide dans la forêt communautaire de Kilum-Ijim : cas de Clausena anisata (Willd.) Hook. f. ex-Benth.
}

Victor Aimé KÉmEuzÉ ${ }^{1}$

Marie Caroline Momo Solefack ${ }^{2}$ Bernard-Aloys NKONGMENECK ${ }^{1}$

Guy DECOCQ ${ }^{3}$

Tafokou JIOFACK ${ }^{1}$

Madeleine JOHNSON ${ }^{1}$

1 Musée écologique du Millénaire Section recherche BP 8038, Yaoundé Cameroun

2 Université de Yaoundé Département de biologie et physiologie végétales BP 337, Yaoundé Cameroun

3 Université de Picardie Jules Verne Chemin du Thil 80025 Amiens France

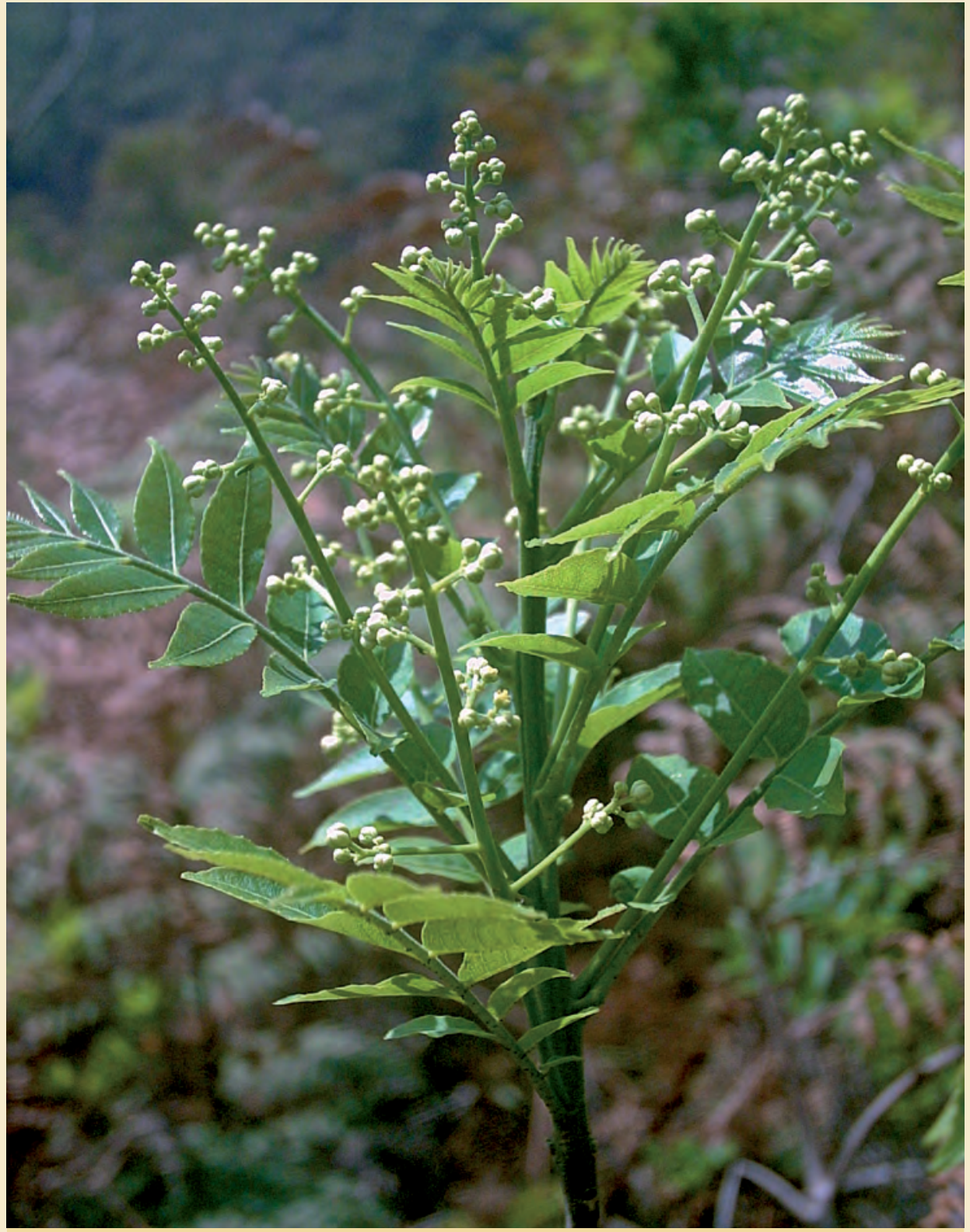

Photo 1.

Plant de Clausena anisata (Willd.) Hook. f. ex-Benth. Photo V. A. Kémeuzé. 
V. A. Kémeuzé,

M. C. Momo SOlefacK,

B.-A. NKongmeneCK, G. DeCocQ,

T. JIOFACK, M. JOHNSON

\section{RÉSUMÉ}

\section{VARIATION ALTITUDINALE DE LA DISTRIBUTION DES PLANTES À ACTIVITÉ INSECTICIDE DANS LA FORÊT COMMUNAUTAIRE DE KILUM-IJIM : CAS DE CLAUSENA ANISATA (WILLD.) HOOK. F. EX-BENTH.}

Dans le souci de contribuer à la valorisation des plantes à activité insecticide, une étude a été réalisée sur la distribution altitudinale de Clausena anisata dans la forêt de Kilum-ljim, dans la province du Nord-Ouest (Cameroun), sur le versant appartenant aux villages de Manchock et Keyon. Grâce à la méthode phytosociologique synusiale intégrée, on constate qu'en dehors de la synusie arborescente Clausena anisata est présent dans toutes les autres synusies. La synusie arbustive contient le plus grand nombre d'individus, avec une fréquence de $40 \%$, suivie de la synusie herbacée et de la synusie sous-arbustive et herbacée haute dont les fréquences respectives sont de $38 \%$ et $22 \%$. Son aire de distribution va de $2100 \mathrm{~m}$ à $2500 \mathrm{~m}$, avec un point de forte densité (175 individus par hectare) entre $2300 \mathrm{~m}$ et $2350 \mathrm{~m}$. Cette espèce présente une bonne régénération dans le milieu. L'absence de jeunes individus autour des pieds adultes a suscité un certain nombre de questions pouvant orienter des recherches futures.

Mots-clés : Clausena anisata, régénération, distribution, insecticide, Kilum-ljim.

\section{ABSTRACT}

\section{RANGE OF DISTRIBUTION BY ALTITUDE OF PLANTS WITH INSECTICIDE PROPERTIES IN THE KILUM-IJIM COMMUNITY FOREST: CLAUSENA ANISATA (WILLD.) HOOK. F. EX BENTH.}

In order to help in developing uses for plants with insecticide properties, a study was carried out on the distribution by altitude of Clausena anisata in the Kilum-Ijim forest located in northwestern Cameroon, on slopes belong. ing to the village communities of Manchock and Keyon. Using an integrated synusial phytosociology method, it was observed that Clausena anisata was present in the tree synusis but also in all other synusia. The highest frequency of individuals ( $40 \%)$, was found in the tree synusis, followed by the herbaceous synusis (38\%) and the understorey and high grass synusis (22\%). Distribution by altitude ranges from $2100 \mathrm{~m}$ to $2500 \mathrm{~m}$ asl, with peak density (175 individuals/ha) between $2300 \mathrm{~m}$ and $2350 \mathrm{~m}$. The species is regenerating well in its natural environment. The absence of young individuals around adult stems has raised a number of questions that offer new research directions.

Keywords: Clausena anisata, regeneration, distribution, insecticide, Kilum-Ijim

\section{RESUMEN}

\author{
VARIACIÓN ALTITUDINAL DE \\ LA DISTRIBUCIÓN DE LAS PLANTAS \\ CON ACTIVIDAD INSECTICIDA EN EL \\ BOSQUE COMUNITARIO DE KILUM- \\ IJIM: CASO DE CLAUSENA ANISATA \\ (WILLD.) HOOK. F. EX BENTH
}

Con el propósito de contribuir al aprovechamiento de las plantas con propiedades insecticidas, se efectuó un estudio sobre la distribución altitudinal de Clausena anisata en el bosque de Kilum-ljim, provincia del Noroeste (Camerún), en la ladera perteneciente a los pueblos Manchock y Keyon. Gracias al enfoque fitosociológico sinusial integrado, se constata que, exceptuando la sinusia arborescente, Clausena anisata está presente en todas las demás sinusias. La sinusia arbustiva engloba el mayor número de individuos, con una frecuencia del $40 \%$, seguida por la sinusia herbácea y la sinusia subarbustiva y herbácea alta, cuyas frecuencias son del $38 \%$ y del $22 \%$, respectivamente. Su área de distribución se escalona entre los 2100 y los $2500 \mathrm{~m}$, con un punto de alta densidad entre los 2300 y los $2350 \mathrm{~m}$. Esta especie presenta una buena regeneración en el medio natural. La ausencia de individuos jóvenes alrededor de pies adultos permitió identificar una serie de cuestiones que podrán orientar futuras investigaciones.

Palabras clave: Clausena anisata, regeneración, distribución, insecticida, Kilum-Ijim 


\section{Introduction}

La lutte contre la faim et celle pour la protection de l'environnement constituent des enjeux auxquels de nombreux pays de la planète s'attellent à trouver des solutions durables.

Plusieurs études ont mis en évidence que l'utilisation des pesticides chimiques en vue d'optimiser le rendement des cultures et leur protection après récolte n'est pas sans conséquence sur la santé et sur la dégradation des sols (Koeler, 2002 ; KÉmeuzé et al., 2006). Du fait de ces multiples problèmes, plusieurs études sont menées, depuis plusieurs années, à la recherche des solutions biologiques capables de combattre efficacement les ravageurs des cultures et les plantes « nuisibles », pour assurer la sécurité alimentaire et garder un environnement sain. Clausena anisata figure parmi ces solutions. Les effets insecticides de cette plante ont déjà été signalés par Letouzey (1963), puis prouvés par Teugwa et al. (2002) au cours des essais réalisés sur Callosobruchus maculatus, parasite du niébé (Vigna unguiculata Walp.). À Oku et dans les villages avoisinants, ses feuilles sont utilisées pour conserver le maïs et le haricot.

La dégradation de la forêt de Kilum-ljim, comme la plupart des écosystèmes des hautes terres de Bamenda, s'intensifie de jour en jour, à cause des multiples activités anthropiques que sont, entre autres, les pâturages, l'artisanat sur bois, l'agriculture itinérante sur brûlis, la chasse et la coupe de bois de chauffe. Ces activités sont la principale cause de la perte de biodiversité. Concernant Clausena anisata (photo 1), la récolte des feuilles sur les individus de grande taille se fait après abattage de ceux-ci. L'intérêt, sans cesse grandissant, porté à cette espèce végétale dans la région pour ses performances insecticides est tel qu'il est souhaitable de sensibiliser la population sur les techniques de récolte durables.

Clausena anisata (Willd.) Hook. f. ex-Benth. a fait l'objet de plusieurs études (notamment : Busı, 2005 ; TEugWA et al., 2002). Le présent travail, qui s'inscrit dans le cadre de la valorisation des plantes insecticides, a pour principal objectif de montrer la distribution, d'estimer la densité des peuplements de Clausena anisata dans la forêt de Kilum-ljim pour permettre une meilleure connaissance de la plante et une utilisation rationnelle et durable de la ressource, dont l'importance dans la région l'expose à une extinction.

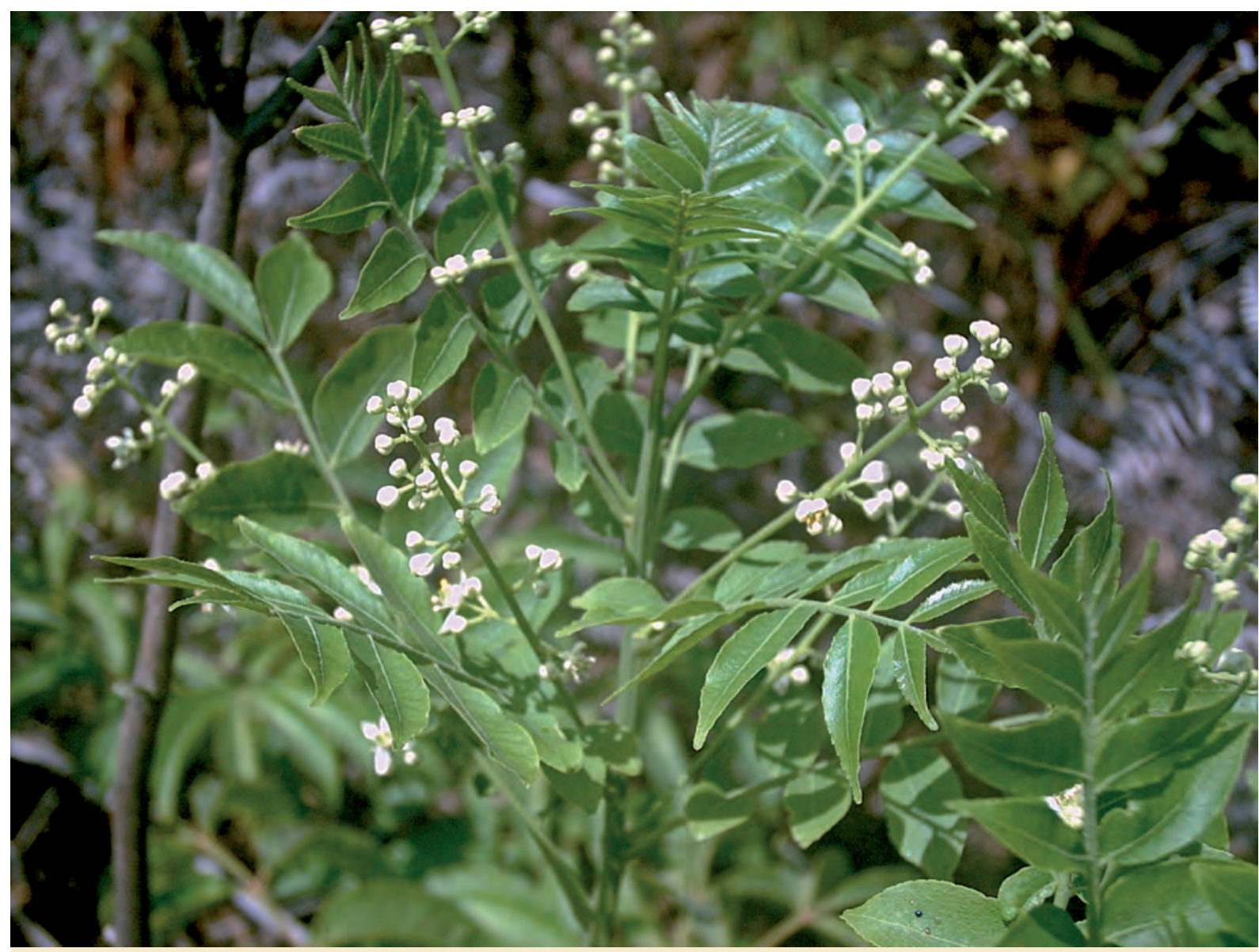

Photo 2.

Clausena anisata en floraison (Willd.) Hook. f. ex-Benth. Photo V. A. Kémeuzé. 


\section{Matériel et méthode}

\section{Description et importance du matériel étudié}

Clausena anisata (rutacée) est un arbuste mesurant en moyenne $2 \mathrm{~m}$ de hauteur mais pouvant atteindre $10 \mathrm{~m}$, et $25 \mathrm{~cm}$ de diamètre. Ses feuilles sont rassemblées à l'extrémité des rameaux et le plus souvent dressées obliquement, longues de 10-30 cm, avec un pétiole de $2 \mathrm{~cm}$ en moyenne, imparipennées, composées d'environ 10 20 folioles alternes ou subopposées de forme elliptique, obliquement ovale ou ovale lancéolée, les supérieures plus grandes que les inférieures. Ces feuilles froissées exhalent une forte odeur désagréable et caractéristique. Son inflorescence (photo 2) est en panicule terminale ou axillaire subterminale, lâche et étroite. Son fruit est une baie monosperme (fruit ayant une seule graine) ellipsoïde de $9 \times 7 \mathrm{~mm}$, blanchâtre, puis rougeâtre, puis bleuâtrenoirâtre (LETOUZEY, 1963, 1985, 1986 ; CHEEK et al., 2000).

Les feuilles de Clausena anisata sont utilisées comme insecticide. Elles permettent, dans la région, de conserver le maïs et son arôme éloigne les moustiques et autres insectes nuisibles dans les concessions.

En outre, dans le patrimoine thérapeutique camerounais, toutes les parties de cette plante sont utilisées dans le traitement de plusieurs maladies, à savoir : la lèpre, les rhumatismes, le paludisme, la stérilité, la syphilis, la gonococcie, la carie dentaire, la gingivite, les maux de tête, les maladies cardiovasculaires et respiratoires, la constipation, la démence, les parasites intestinaux, la gastro-entérite, l'anémie, etc. (OJEWOLE, 2002 ; TEKLEHAYMANOT, GIDAY, 2007). M. David Tchinda, tradithérapeute du village de Keyon, lui reconnaît un pouvoir magique très puissant. Selon lui, les feuilles de Clausena anisata seraient capables d'empêcher le sommeil chez un être humain lors d'un voyage. Elles sont utilisées dans le traitement des cas de démence par celui-ci.

Les modes d'utilisation des parties de cette plante et d'administration des dérivés varient avec la maladie. Toutefois, on peut noter que la plupart des décoctés obtenus sont buvables. Aucune étude de toxicité n'est connue à ce jour.

\section{Site de l'étude}

Le massif de Kilum-ljim, communément appelé mont Oku, est une forêt communautaire située dans la province du Nord-Ouest, dans les départements de Bui et Boyo. Son point culminant possède les coordonnées $6^{\circ} 12^{\prime} \mathrm{N}$ et $10^{\circ} 32^{\prime} \mathrm{E}$ tandis que le lac Oku se localise à $6^{\circ} 12^{\prime} \mathrm{N}$ et $10^{\circ} 27^{\prime} \mathrm{E}$. La forêt dense sempervirente submontagnarde du mont Oku est entièrement entourée par la savane de basse altitude et les terres agricoles de moyenne altitude.

La forte pluviosité sur le massif a créé un réseau dense de rivières qui vont vers la Katsena (Benoué) ou vers le Mbam (Sanaga). Tous les cours d'eau sont permanents à cause de la forêt sempervirente ; ils ont pour nom Wum, Mankon, qui coulent vers la plaine de Ndop, Mugom, Jonka et Mentor, qui coulent vers la Menchum, tandis que Kimbi Sunta et Mintua vont vers la Katsena.

La zone est un volcan éteint, comme en témoigne le lac de cratère qui occupe la caldeira. Les formations géologiques qu'on y rencontre sont faites de basalte, trachyte, rhyolite, gneiss migmatique et granite. Les sols du massif de Kilum-ljim ont été classés par HAWKINS et BRUNT (1965) dans la catégorie des sols ferrallitiques humifères. Ces sols ont une teneur en matière organique élevée. La partie superficielle du sol est habituellement de couleur noire, tandis que les couches inférieures dans le profil sont brun rougeâtre sombre, passant au rouge sombre ou rouge jaunâtre en profondeur. Ce sont des sols argileux, l'argile dominante étant la gibbsite, mais ils ont l'appa- rence du terreau, du fait de leur contenu en matière organique élevé. Ils tendent à avoir une structure granulaire près de la surface.

Le climat est de type tropical humide, à pluviosité unimodale, caractérisé par une longue saison des pluies (mars-novembre) et une courte saison sèche (novembremars). Il subit l'effet de l'altitude, qui se traduit par une grande nébulosité, de fortes précipitations et des températures basses. L'indice pluviométrique varie de $1800 \mathrm{~mm}$ dans la plaine à $3 \mathrm{~m}$ en altitude. La valeur moyenne des maxima oscille entre $22^{\circ} \mathrm{C}$ à partir de $1800 \mathrm{~m}$ et $16^{\circ} \mathrm{C}$ au sommet (minima de $13^{\circ} \mathrm{C}$ à $9{ }^{\circ} \mathrm{C}$ respectivement). Les précipitations occultes, brume, brouillard et rosée, sont abondantes.

La forêt de Kilum-ljim est une forêt naturelle dense humide de montagne, couvrant de l'ordre de 20000 ha, qui est située dans la province du NordOuest (hautes terres de Bamenda). C'est l'une des forêts de montagne résiduelles parmi les plus significatives d'Afrique centrale. Le mont Kilum, à 3011 m d'altitude, et la crête ljim constituent un centre d'endémie important et un " point chaud " (hotspot) pour la conservation de la biodiversité. Il existe onze types de végétation principaux dans cette forêt, avec entre autres des forêts de Podocarpus latifolius, Prunus africana, Rapanea melanophloeos aux plus hautes altitudes, des forêts à Adenocarpus mannii, Hypericum revolutum et Gnidia glauca, des forêts de montagne, des forêts secondaires, des formations herbeuses régulièrement brûlées par les pasteurs. La forêt contient également de nombreuses espèces végétales et animales endémiques (notamment des oiseaux et des amphibiens). Cette forêt revêt un rôle important dans la régulation des eaux et, en outre, elle a une valeur culturelle et spirituelle essentielle pour la population locale. Les travaux de l'étude ici présentée se sont déroulés dans les secteurs des villages de Manchock et Keyon. 


\section{Méthode}

C'est la méthodologie phytosociologique qui a été retenue et suivie sous son approche synusiale " intégrée » (GILLET, 2000 ; DeCOCQ, 2002). Cette méthode s'est révélée être la mieux adaptée aux végétations forestières tropicales, car elle permet ultérieurement une modélisation systémique qualitative de la dynamique végétale. Afin de contribuer à une meilleure connaissance de la dynamique de la forêt de Kilum-Ijim, un inventaire global allant des herbes aux arbres a été effectué.

L'échantillonnage s'est fait par des quadrats suivant le gradient topographique altitudinal, de $2108 \mathrm{~m}$ à $3011 \mathrm{~m}$, et l'homogénéité du substrat, en s'efforçant d'être représentatif des diverses conditions naturelles et anthropiques.

La taille des relevés était de $40 \mathrm{~m}$ $\times 40 \mathrm{~m}$, soit $1600 \mathrm{~m}^{2}$, pour les synusies arborescentes $\left(A_{1}\right.$ : arbres de $30 \mathrm{~m}$ et plus ; $A_{2}$ : arbres de 10-30 m) de même que pour les synusies arbustives $\left(a_{1}\right.$ : arbustes de 2-10 $\mathrm{m} ; \mathrm{a}_{2}$ : arbustes de $40 \mathrm{~cm}-2 \mathrm{~m}$ ) et pour les herbacées hautes ( $\mathrm{H}$ : herbacées de $40 \mathrm{~cm}-2 \mathrm{~m}$ ),

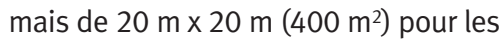
synusies herbacées ( $h$ : herbacées de moins $40 \mathrm{~cm}$ ). Toutes les données ont été ramenées à l'hectare afin de comparer les différentes populations par des analyses statistiques. Concernant Clausena anisata, une marque a été faite sur les individus mesurés pour éviter les erreurs de comptage. Toutes les plantules présentes dans les relevés ont également été dénombrées afin de mieux appréhender le mode de régénération de la population. Dans la présente étude, seuls les individus vivants ont été pris en compte.

Pour une meilleure appréciation du fonctionnement des populations de Clausena anisata, des observations complémentaires sur la multiplication végétative de cette espèce ont été réalisées. Elles ont consisté en un comptage du nombre de rejets par souche. Seule la circonférence du plus grand rejet a été notée pour matérialiser l'individu.

\section{Résultats et discussion}

\section{Analyse des populations de Clausena anisata (Willd.) Hook. f. ex-Benth.}

L'analyse des populations ligneuses, selon Onana et Devineau (2002), peut se réaliser autour de deux éléments principaux que sont, d'une part, la répartition des individus en classes de taille ou de diamètre, comme indicateur indirect du niveau d'équilibre des classes d'âge et comme témoin des phases vécues par la population en termes de perturbation ou de régénération, et, d'autre part, l'intensité de la régénération, symbole du renouvellement à terme de la population. (Un possible troisième élément, le mauvais état général des peuplements, n'a pas pu être pris en compte dans la présente étude.)

Dix relevés de structure de population ont été effectués. La figure 1 montre que toutes les différentes classes d'âge sont représentées, avec une dominance de jeunes individus. Cette abondance de plantules indique une bonne régénération de l'espèce. La faible représentativité des individus de grande taille peut s'expliquer soit par la pression exercée sur la ressource dans le milieu - selon les guides de terrain locaux, au moment de la récolte des feuilles utilisées pour la protection des produits vivriers, certains individus de grande taille sont étêtés (photo 3) pour faciliter la collecte des feuilles -, soit par l'ouverture de la canopée, car Clausena anisata est une plante héliophile et la réduction du flux solaire ralentirait son développement.

\section{Chorologie de Clausena anisata dans le milieu}

Lors des différentes observations, on a noté une rareté des plantules au pied des individus adultes. Cette observation a permis d'émettre l'hypothèse selon laquelle les fruits de Clausena anisata seraient consommés par certains animaux rejetant les graines au loin et assurant ainsi sa dissémination dans le milieu ; et cela en sachant, d'après quelques personnes-ressources, que les graines de cette espèce seraient appréciées par des oiseaux et des rats. Ces connaissances restent empiriques car seul un suivi rigoureux peut permettre une meilleure appréciation des différents acteurs responsables de la chorologie de cette espèce végétale.

Toutefois, l'hypothèse d'un phénomène d'allélopathie reste certes envisageable, mais difficile à justifier dans le cadre de cette étude.

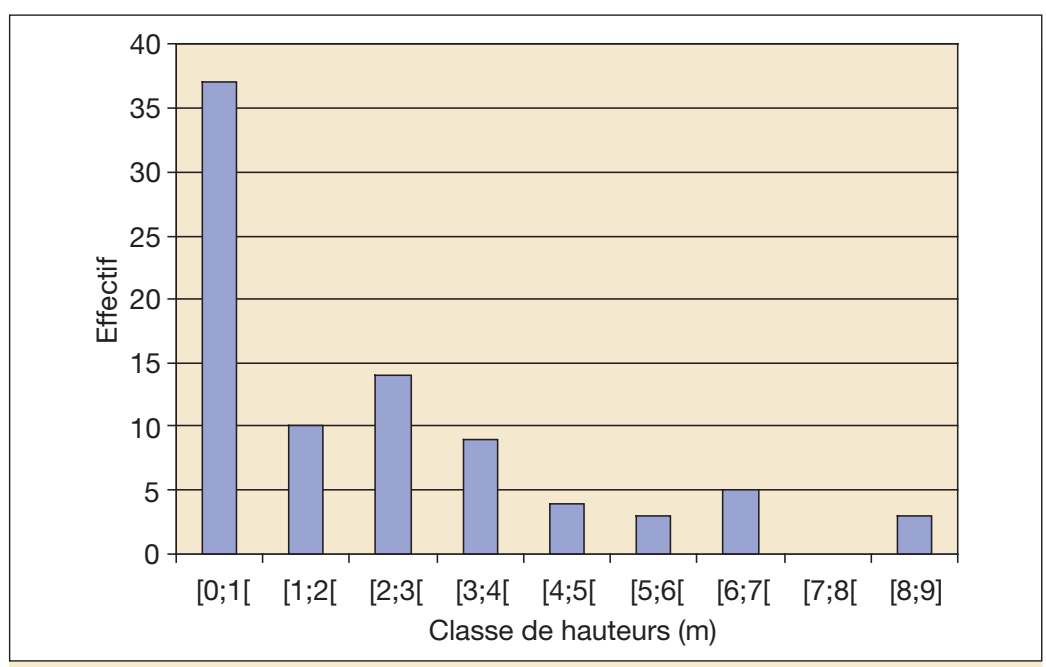

Figure 1.

Histogramme moyen de la distribution des individus par classe de hauteur chez Clausena anisata. 


\section{Structure verticale de la population}

En dehors de la synusie arborescente, Clausena anisata est distribuée dans toutes les autres synusies. La synusie arbustive contient le plus grand nombre d'individus, avec une fréquence de $40 \%$, suivie de la synusie herbacée et de la synusie sousarbustive et herbacée haute, dont les fréquences respectives sont de $38 \%$ et 22 \% (figure 2). Son absence dans la synusie arborescente est due au fait que sa hauteur n'excède pas dix mètres au Cameroun (Letouzey, 1963).

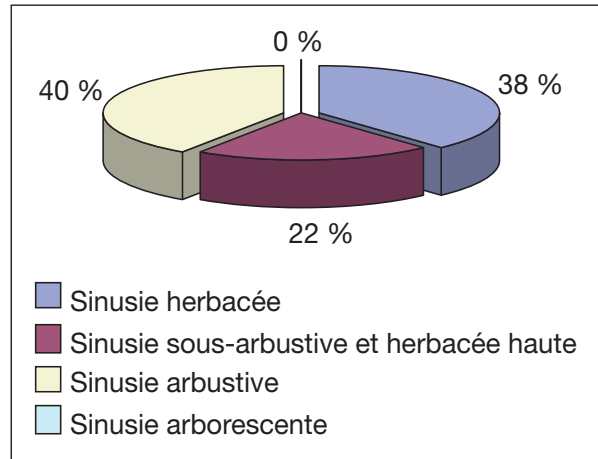

Figure 2.

Fréquences de Clausena anisata dans les différentes synusies.

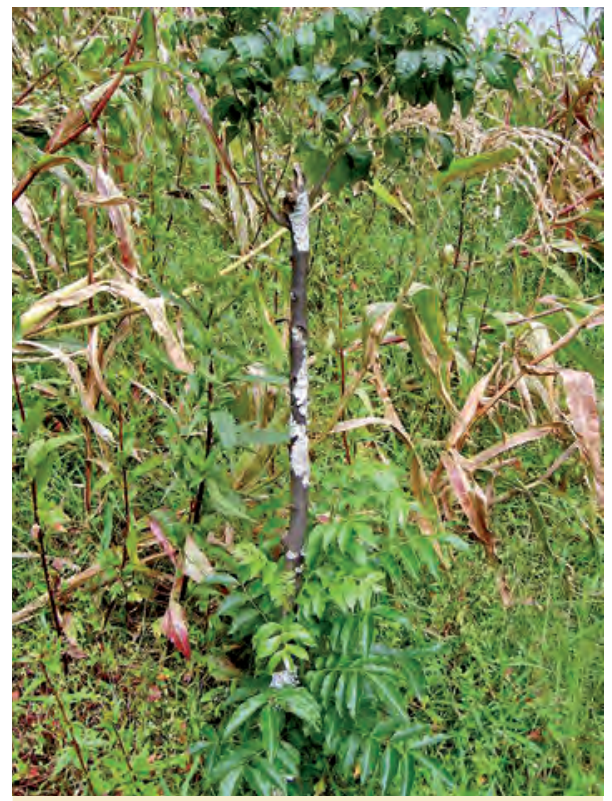

Photo 3.

Clausena anisata (Willd.) Hook. f. ex-Benth. étêtée se trouvant dans une plantation vivrière. Photo V. A. Kémeuzé.

\section{Distribution altitudinale des populations de Clausena anisata}

Sur la figure 3, la courbe en cloche du graphique montre l'aug. mentation de la densité de la population de Clausena anisata en fonction de l'altitude. Elle culmine entre 2300 et $2350 \mathrm{~m}$ (175 individus par hectare), altitude à partir de laquelle elle diminue pour s'annuler autour de 2500 m, ce qui coïncide avec l'apparition de Podocarpus latifolius dans le milieu. Les faibles proportions dans de basses altitudes peuvent s'expliquer par la proximité des plantations. Ces endroits font l'objet d'une exploitation intense des populations riveraines. Ce résultat montre la limite de la distribution altitudinale de cette espèce au Cameroun. Toutefois, des études supplémentaires doivent être menées dans les autres parties de la forêt de Kilum-ljim pour corroborer les résultats de ce travail, car en Afrique de l'Est sa présence est mentionnée à 3000 m d'altitude (BusIA, 2005).

\section{Multiplication végétative}

Au stade adulte, à la suite d'un traumatisme, une plante peut soit mourir, soit émettre des rejets dont l'importance varie avec le rythme des traumatismes subis. Le tableau I donne la moyenne des rejets par relevé phytosociologique.

À la lecture du tableau I, on peut constater que les relevés $R_{1}, R_{3}, R_{8}$ et $R_{9}$ subissent une pression importante de la part des populations qui abattent systématiquement les grands individus pour récolter leurs feuilles. L'absence de rejets dans les autres relevés peut être due soit à l'absence de l'espèce dans le milieu, soit au fait que les traumatismes ont entraîné la mort de ces individus.

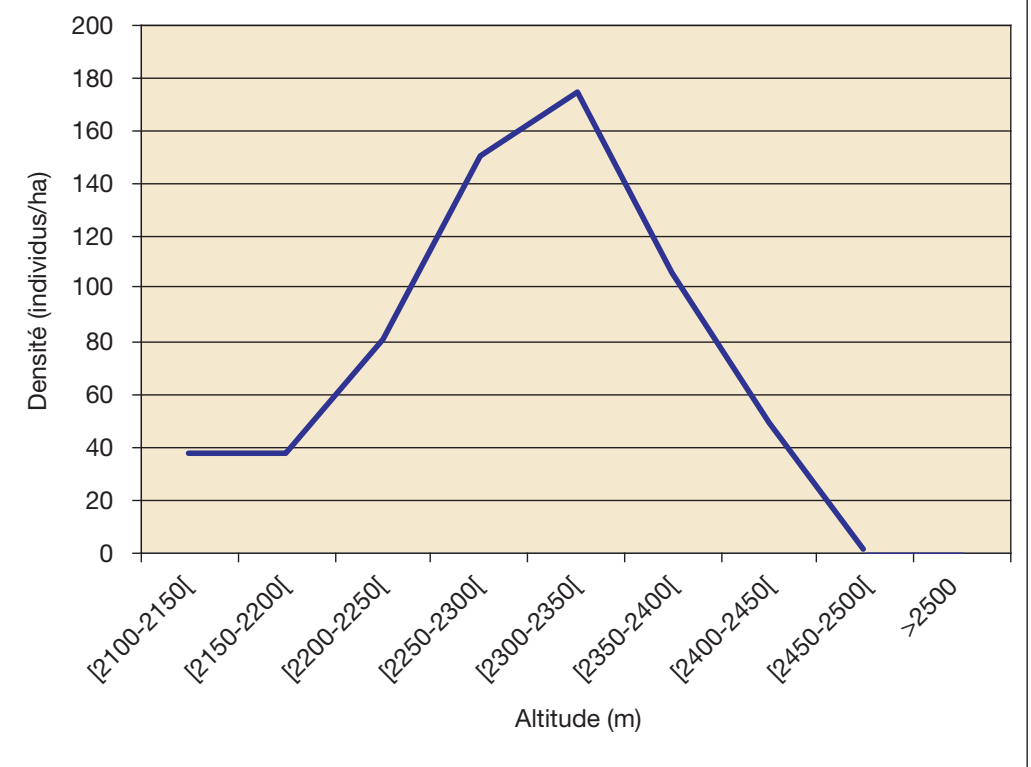

Figure 3.

Distribution des populations de Clausena anisata (Willd.) Hook. f. ex-Benth. en fonction de l'altitude dans les villages de Manchock et Keyon. 
Tableau I.

Évolution moyenne des rejets de $C$. anisata par transect.

\begin{tabular}{|c|c|c|c|c|c|c|c|c|c|c|}
\hline Relevé & $\mathrm{R}_{1}$ & $\mathrm{R}_{2}$ & $R_{3}$ & $\mathrm{R}_{4}$ & $R_{5}$ & $R_{6}$ & $R_{7}$ & $\mathrm{R}_{8}$ & $\mathrm{R}_{9}$ & $\mathrm{R}_{10}$ \\
\hline Altitude (m) & 2108 & 2495 & 2161 & 2136 & 2400 & 2345 & 2248 & 2251 & 2344 & 2502 \\
\hline Effectif & 9 & 0 & 6 & 3 & 8 & 2 & 24 & 24 & 15 & 0 \\
\hline Moyenne de rejets par souche & 1 & 0 & 0,9 & 0 & 0 & 0 & 0 & 1,45 & 0,14 & 0 \\
\hline
\end{tabular}

\section{Conclusion}

Cette étude bien qu'étant partielle apporte sa propre contribution à la valorisation de la flore camerounaise en général et celle de la forêt de Kilum-ljim en particulier, appartenant à la région montagnarde du Cameroun. Clausena anisata présente une bonne régénération dans le milieu. Sa densité croît avec l'altitude. Elle atteint son maximum entre $2300 \mathrm{~m}$ et 2350 m, altitude à partir de laquelle elle diminue pour s'annuler autour de 2500 m. Face aux enjeux que constituent la protection de l'environnement et la sécurisation alimentaire, l'énorme pression anthropique que subit cette ressource dans la région est un signal fort qui est un appel à une prise de conscience des populations locales pour une gestion harmonieuse et durable.

\section{Remerciements}

Nous remercions tous nos guides de terrain et tous les Fmi (Forest Management Institution) de l'arrondissement de Oku.

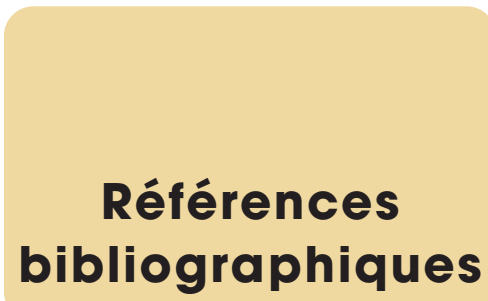

BUSIA K., 2005. Monograph of Clausena anisata (Willd.) Hook. F. ex Benth. Journal of Health, Social and Environmental Issues, 6 (2) : 19-23.

CHEEK M., ONANA J.-M., POLLARD B. J., 2000. The plants of Mount Oku and the ljim ridge, Cameroon. A conservation checklist. Kew, RoyaumeUni, Royal Botanic Gardens, 211 p.

DECOCQ G., 2002. Patterns of plant species and community diversity at different organization levels in a forested riparian landscape. Journal of Vegetation Science, $13:$ 91-106.

GILLET F., 2000. La phytosociologie synusiale intégrée. Guide méthodologique. Institut botanique, Université Neuchâtel, Document du Laboratoire d'écologie végétale, 1, 68 p.

HAWKINS P., BRUNT M., 1965. The soils and ecology of West Cameroon. Rome, Italie, Fao, 2 vol., 516 p.

KÉMEUZÉ V. A., NKONGMENECK B. A., DELNEUF M., FONGNZOSSIE F. E., JIOFACK T., 2006. Problématique de l'utilisation des herbicides et lutte contre la désertification dans l'arrondissement de Mbé (AdamaouaCameroun). Biosciences Proceedings, vol. 12.

KOEHLER C., 2002. Les pesticides chimiques, le piège se referme. Agridoc, art. $\mathrm{n}^{\circ} 76, \mathrm{p}$. II-III.

LETOUZEY R., 1963. Flore du Cameroun $n^{\circ} 1$ : Rutacées, Zygophyllacées, Balanitacées. Paris, France, Muséum national d'histoire naturelle, $174 \mathrm{p}$.
LETOUZEY R., 1985. Notice de la carte phytogéographique du Cameroun au 1/500 000. M-SM : Région afro-montagnarde et étage submontagnard. Toulouse, France, Institut de la carte internationale de la végétation, p 27-62.

LETOUZEY R., 1986. Manual of forest botany, tropical Africa. Nogent-surMarne, France, Centre technique forestier tropical, vol. 2A et 2B, $461 \mathrm{p}$.

OJEWOLE J. A., 2002. Hypoglycaemic effect of Clausena anisata (Willd.) Hook. methanolic root extract in rats. Journal of Ethnopharmacolgy, 81 (2) : 231-237.

ONANA J., DEVINEAU J.-L., 2002. Afzelia africana Smith ex Persoon dans le Nord-Cameroun. État actuel des peuplements et utilisation pastorale. Revue d’Élevage et de Médecine Vétérinaire des Pays Tropicaux, 55 (1) : 39-45.

TEKLEHAYMANOT T., GIDAY M., 2007. Ethnobotanical study of medicinal plants used by people in Zegie Peninsula, Northwestern Ethiopia. Journal of Ethnobiology and Ethnomedicine, 3 : 12.

TEUGWA M. C., PIAM G., TANE P., AMVAM ZOLLO P. H., 2002. Activité insecticide des extraits d'Ageratum houstonianum, de Clausena anisata et de Croton macrostachyus sur la bruche du niébé (Vigna unguiculata Walp.). FoodAfrica, p. 1-4. 